American Journal of Pharmacology and Toxicology 2 (4): 159-163, 2007

ISSN 1557-619X

(C) 2007 Science Publications

\title{
Sesame Oil as a Protective Agent Against Doxorubicin Induced Cardio Toxicity in Rat
}

\author{
${ }^{1}$ Kaneez Fatima Shad, ${ }^{2}$ Suhail Al-Salam and ${ }^{3}$ Alaaeldin A. Hamza \\ ${ }^{1}$ Karachi University, Pakistan \\ ${ }^{2}$ Department of Pathology, Faculty of Medicine and Health Science, UAE University UAE \\ ${ }^{3}$ Department of Biology, UAE University UAE
}

\begin{abstract}
The therapeutic value of doxorubicin (DXO) as anticancer antibiotic is limited by its cardiotoxicity. This study aimed to investigate the protective effect of sesame oil against DXO-induced cardiotoxicity and oxidative stress. The administration of DXO $(20 \mathrm{mg} / \mathrm{kg})$ to Wister male rats, increased serum aspartate aminotransferase and creatine kinase activities (as cardiotoxicity indices) as well as cardiac malondialdehyde and protein carbonyl contents and catalase activity (as markers of oxidative stress). The cardiotoxicity damage in DOX treated rats was accompanied by histo pathological changes such as necrosis and apoptosis in cardiac tissues. These biochemical and histological disturbances were effectively attenuated on pretreatment with sesame oil $(5 \mathrm{ml} / \mathrm{kg})$. These data suggest that sesame oil may be a suitable cardio protector during DOX treatment.
\end{abstract}

Key words: Doxorubicin, Cardio toxicity, Oxidative stress, Protective effect of Sesame oil

\section{INTRODUCTION}

Doxorubicin ${ }^{[}$Adriamycin,

(DOX)],

an anthracycline antibiotic, is widely used as effective antineoplastic agent in the treatment of a variety of malignancies, including lymphoma, leukemia and solid tumors. However, DOX causes various toxic effects, the commonest of which is the dose-dependent cardiotoxicity ${ }^{[1]}$. Cardiotoxicity of DOX is mediated by the formation of free radicals and oxidative damage to cellular components ${ }^{[2]}$. DOX is enzymatically reduced to the DOX semiquinone radical. This semiquinone radical directly transfers its electron to molecular oxygen, generating a superoxide and hydrogen peroxide $^{[3]}$. Because of free radical plays important roles in DOX-induce cardiotoxicity, it is logical to consider antioxidants as primary potential therapeutic agent to prevent such toxic effect. In fact, several compounds with antioxidant properties have been investigated with some degree of success. These include p-coumaric acid ${ }^{[4]}$, garlic acid ${ }^{[2]}$, erdosteine ${ }^{[5]}$ and carvedilol ${ }^{[1]}$.

Sesame Oil (SO) is extract from the plant Sesamum indicum (HS), family: Pedaliacea $\mathrm{e}^{[6]}$. SO offers protection over blood pressure, lipid profiles and lipid peroxidation in hypertensive patients ${ }^{[7]}$. SO attenuates oxidative stress and multiple organ failure triggered by endotoxin lipopolysaccharide in rats $^{[8]}$. Sesamin and sesaminol are the major phenolic constituents of SO which have been reported to possess a broad spectrum of pharmacological effects including antimutagenic ${ }^{[9]}$,

antioxidant ${ }^{\left[10,11, \hat{1}_{2}\right]}$,antihypertensive ${ }^{[11]}$, antiinflammatory ${ }^{[12]}$ and antithrombotic ${ }^{[13]}$.

This investigation was set to evaluate the protective effects of SO against DOX-induced cardiotoxicity in male rats and to study the mechanisms underlying these effects.

\section{MATERIALS AND METHODS}

Chemicals: The pure SO was purchased from Amoy Food Ltd. (Birmingham, England). DOX (Adriblastina) was purchased from Pharmacia Italia S.p.A. (Nerviana, Italy). USA. Thiobarbituric acid, Folin's reagent, epinephrine, SOD enzyme, $\mathrm{H} 2 \mathrm{O} 2$ and bovine albumin were obtained from Sigma Chemical Co. (St. Louis, $\mathrm{MO}$ ). The reagent kit for aspartate aminotransferase enzyme activity was purchased from BioMerieux (RCS Lyon, France). All other chemicals were obtained from local commercial suppliers.

Animals: Adult male albino rats $(150-200 \mathrm{~g})$ of the Wistar strain were obtained from the Animal House, UAE University, U.A.E. They were maintained on standard pellet diet and tap water ad libitum and were kept in polycarbonate cages with wood chip bedding under a $12 \mathrm{hr}$ light/dark cycle and room temperature 22-

Corresponding Author: Professor, Dr Kaneez Fatima Shad, Panjwani Center for Molecular Medicine and Drug Research, Karachi University, Karachi 75270, Pakistan 
$24^{\circ} \mathrm{C}$. Rats were acclimatized to the environment for one-week prior to experimental use. This study was approved by the Animal Ethics Committee, UAE University.

Treatment regime: DOX solution was freshly prepared, protected from light in a saline solution. SO was given orally by gavage at a volume of $5 \mathrm{ml} / \mathrm{kg}$ body weight. Rats were randomly divided into four groups (five rats each) and were subjected to the following treatments: In the DOX-treated group, animals were given a single intraperitoneal dose of DOX (20 mg/kg b.wt.), which has been used previously to induce heart toxicity in various animal species ${ }^{[5]}$. Animals of the third group were fed SO extract for 27 days and heart toxicity induced by injected the same single dose DOX after three weeks of SO administration. The fourth group was treated with SO daily for 27 days. The control group was treated with distilled water ( $5 \mathrm{ml} / \mathrm{kg}$ b.wt.), for 27 days and injected with saline after three weeks of water administration. After 6 days of DOX and 27 days of oil and vehicle solution administrations, blood and hearts were collected from all groups.

Sample preparation: Following diethyl ether anesthesia, blood was collected from the retro-orbital plexus. Following sacrifice hearts were removed and weighed. The organ weight/body weight ratio x 100 was calculated and expressed as a relative organ weight. For histopathological examination, half of the heart was immediately fixed in $10 \%$ buffered formalin. For biochemical determination, another half of the heart was homogenized in ice-cold $\mathrm{KCl}(150 \mathrm{mM})$. The ratio of tissue weight to homogenization buffer was 1:10. From the latter, suitable dilutions for determination of the levels of protein carbonyl (P.carbonyl), malondialdehyde (MDA), total proteins and activity of catalase (CAT) were prepared in suitable different buffers. To obtain serum, blood was collected in centrifuge tubes and centrifuged in a refrigerated centrifuge $\left(4^{\circ} \mathrm{C}\right)$ at 3000 r.p.m. for $20 \mathrm{~min}$.

Biochemical Assays and Histopathology: Activity of aspartate aminotransferase (AST) was determined in serum according to the method described by ${ }^{[14]}$ and the assay was performed using BioMerieux reagent kit and following its instruction manual. Whereas, determination of MDA in biological materials, as described in $^{[15]}$, was based on its reaction with thiobarbituric acid (TBA) to form a pink complex with absorption maximum at $535 \mathrm{~nm}$.

CAT activity was determined by measuring the exponential disappearance of $\mathrm{H}_{2} \mathrm{O}_{2}$ at $240 \mathrm{~nm}$ and expressed in units/mg of protein as described by ${ }^{[16]}$.
The total antioxidant capacity in heart was evaluated using ferric reducing antioxidant power (FRAP) assay. The FRAP assay was determined according to the method described by ${ }^{[17]}$. The FRAP assay measures the change in absorbance at $593 \mathrm{~nm}$ due to the formation of a blue colored ferrous- tripyridyltriazine complex from colorless oxidized ferric form by the action of electron donating antioxidants.

Cardiac P.carbonyl contents were determined according to method of ${ }^{[18]}$. This method is based on spectrophotometric detection of the reaction of 2,4dinitophenylhydrazine with P.carbonyl to form protein hydrazones at $370 \mathrm{~nm}$. The results were expressed as nmol of carbonyl group per milligram of protein with molar extinction coefficient of $22000 \mathrm{M} / \mathrm{cm}$.

The total protein content of heart was determined according to the Lowry method as modified by ${ }^{[19]}$. Absorbance was recorded using Shimadzu recording spectrophotometer (UV-160) in all measurements.

For the histological examinations, pieces of hearts were fixed in $10 \%$ neutral phosphate-buffered formalin, dehydrated in ethanol, cleared in xylene and embedded in paraffin Five-micrometer sections were stained with Hematoxylin and Eosin. The sections were examined under a Leica DMRB/E light microscope.

Statistical Analysis: SPSS (version 10) statistical program (SPSS Inc., Chicago, IL, USA) was used to carry out a one-way analysis of variance (ANOVA) on our data. When significant differences by ANOVA were detected, analysis of differences between the means of the treated and control groups were performed by using Dunnett's t test.

\section{RESULTS AND DISCUSSION}

Change in weight of heart and serum marker of heart damage: The relative weight of the organ is the percent of organ weight to the body weight. Treatment with DOX significantly $(\mathrm{P}<0.001)$ decreased relative

Weight of the Heart (Figure 1a). However, there was No significant change in relative weight of heart animal groups treated with $\mathrm{SO}$ alone or before DOX treatment. After treatment with DOX, The levels of serum marker of heart damage, AST significantly $(\mathrm{P}<0.001)$ increased compared to the control after. The treatment with SO alone did not show any significant changes in the AST activity, (Figure 1b). While the treatment with SO before DOX treatment attenuated this increase in AST level. 


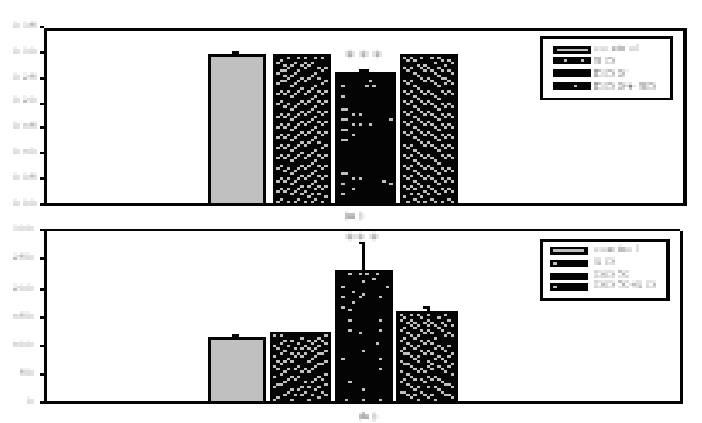

Fig. 1: Effect of SO on (a) relative weight of heart (\%) and serum AST activity (u/dl) (b) in control-and DOX -treated rats. Each column represents the mean $\pm \mathrm{SE}$, for five rats in each group. *** $\mathrm{P}<0.001$ vs $\mathrm{C}$. Role of $\mathrm{SO}$ on oxidative stress : DOX treated rats showed a significant increase in the markers of oxidative stress, P.carbonyl $(\mathrm{P}<0.01)$ (Figure 2a) and MDA $(\mathrm{P}<0.05)$ (Figure $2 \mathrm{~b})$ and in CAT activity $(\mathrm{P}<0.01)$ (Figure $3 \mathrm{a})$ and FRAP content $(\mathrm{P}<0.05)$ (Fig 3b)

Histopathological effects of Doxorubicin ${ }^{[}$Adriamycin, (DOX)]: Figure 4 shows the light micrograph of control heart showing normal architecture. Severe degenerations of the myofibrils, with focal necrosis, vacuolated cytoplasm were clearly seen in the DOXtreated group. DOX intoxication also induced eosinophilic cytoplasm, focal hemorrhage and with inflammatory cell infiltrations. Animals pretreated with SO showed better-preserved appearance of cardiac muscle fibers with slight degeneration and some leukocyte infiltration. Cardiac section of SO treated rats shows normal structure.
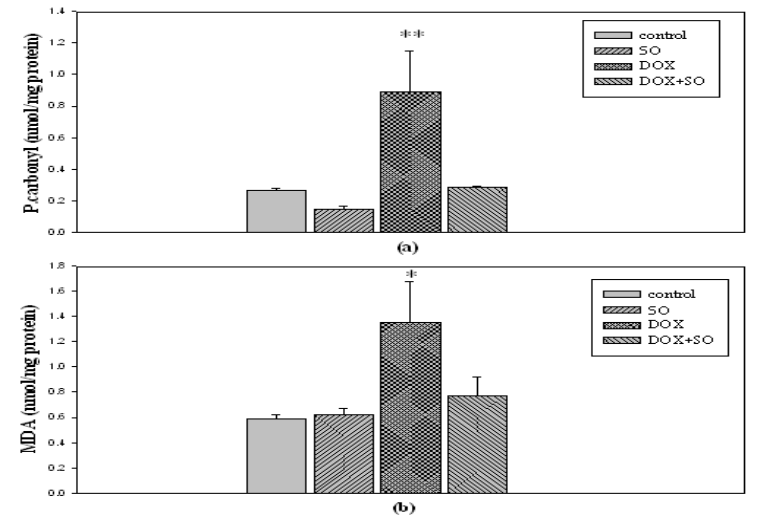

Fig. 2: Effect of SO on cardiac (a) P.carbonyl \& (b) MDA levels (nmol / mg protein) in control- \& DOX- treated rats. Each column represents the mean $\pm \mathrm{SE}$, for five rats in each group. $* \mathrm{P}<$ $0.05 ; * * \mathrm{P}<0.01$ vs. $\mathrm{C}$
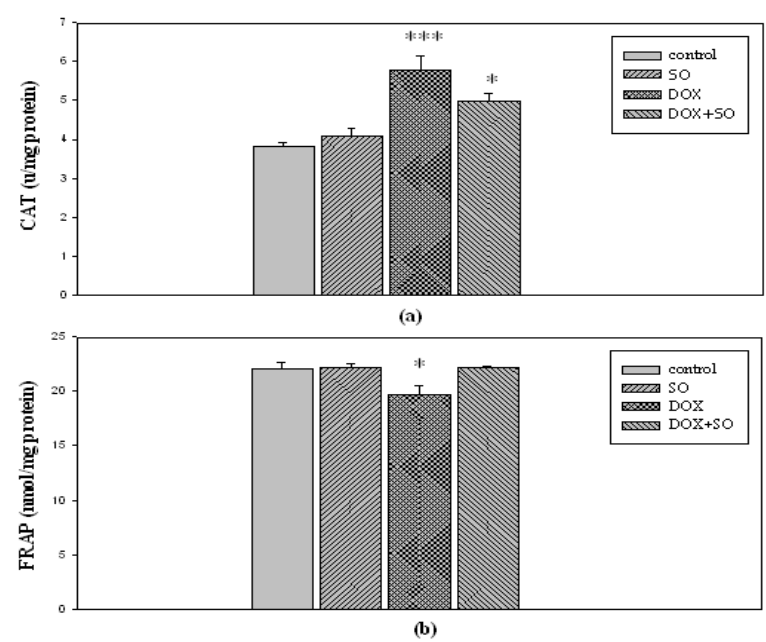

Fig. 3: Effect of SO on cardiac (a) CAT activity (u / mg protein) and (b) FRAP content (nmol / $\mathrm{mg}$ protein) in control- and DOX- treated rats. Each column represents the mean $\pm \mathrm{SE}$, for five rats in each group. ${ }^{*} \mathrm{P}<0.05 ; * * * \mathrm{P}<$ 0.001 vs. control.

There is a significant decrease in FRAP assay $(\mathrm{P}<0.05)$ (Figure $3 b$ ) in heart. While SO pretreatment completely prevented the elevation in P.carbonyl and MDA as well as the depletion in FRAP. Rats received SO alone did not show any changes in these oxidative stress markers. The treatment with DOX induced elevation $(\mathrm{P}<0.001)$ in cardiac CAT activity and the treatment with SO attenuated this effect $(\mathrm{P}<0.05)$ (Figure $3 \mathrm{a})$.

The protective effect of SO on cardiomyopathy of DOX was investigated in male Wister rats. To our knowledge, this is the first study that evaluates the protective effects of $\mathrm{SO}$ against myocardial damage induced by DOX in experimental animals. In this report, a single dose of DOX (20 mg/kg b wt) induced severe heart toxicity in adult male rats. Treatment with DOX has been shown to cause cardiomyopathy in various animal species ${ }^{[1,20,5]}$. The present results showed a significant reduction in the relative weight of heart and an increase in serum AST activity (a reliable biomarker of cardiac injury) after treatment with DOX. This DOX-induced myocardial damage was also confirmed by myocardial histopathological changes (Myocyte necrosis, degeneration and inflammatory cell infiltrations) similar to those reported in other studies $^{[2,5,20] \text {. }}$

In this study, DOX-treated animals showed an elevation in myocardial MDA and P.carbonyl levels and depletion in total antioxidant capacity (FRAP) versus the control group, which are important indices of oxidative damage. A possible explanation for the 
increased MDA and P.carbonyl concentrations might be the increased generation of reactive oxygen species (ROS) in DOX-induced tissues. ROS react with cardiac myocyte membrane and cause oxidative damage to cellular lipids, proteins and DNA and cause lipid and protein peroxidation and DNA fragmentation ${ }^{[21]}$. The role of oxidative stress, the elevation of oxidative macromolecules and the disturbance in antioxidant defenses in heart of DOX treated rats has been observed in previous studies ${ }^{[1,5,22]}$. The anthracycline ring structure of DOX has been shown to induce both enzymatic and non-enzymatic single-electron redox cycle liberation of ROS from molecular oxygen ${ }^{[3]}$.

Although cardiac total antioxidant capacity decreased in this study, CAT activity increased in DOX treated rats. This indicates the massive production of hydrogen peroxide in the myocardium and the significant role of hydrogen peroxide in DOX-induced cardio toxicity. The elevation in cardiac CAT activity has been reported in the previous studies ${ }^{[22]}$ in male Wister rats treated with DOX. In fact, DOX functions as an anticancer drug through oxidative DNA damage and apoptosis by the DOX- induced direct hydrogen peroxide generation $^{[23]}$. The redox-cycle of DOX in the presence of cytochrome P450 reductase and NADPH was considered as the major source of hydrogen peroxide $^{[3,23]}$.

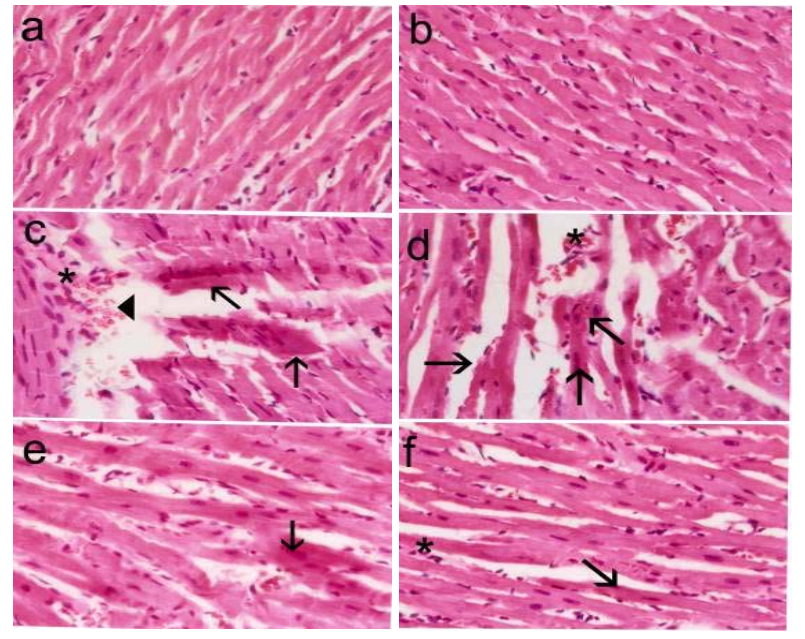

Fig: 4 Photomicrograph of the heart of control (a) and SO- treated rats (b) showing the normal myofibrillar structure with striations and branched appearance. Hearts of DOX- treated rats (c \& d) showing focal necrosis of muscle fibers with eosinophilia in the cytoplasm (arrows). Heart of DOX-treated rats also showing destruction of muscle fibers with Focal hemorrhage (arrowheads) and inflammatory cell infiltrations (stars). Group of rats pretreated with SO (e) \& (f) before DOX exhibiting relatively normal myocardial cells with vascular dilatation and moderate degeneration of some fibril cells. (H\&E, X 200).

Recently, much attention has been focused on the protective effects of antioxidants and naturally occurring substances against DOX-induced cardiotoxicity ${ }^{[4,2]}$. Administration of SO before DOX treatment clearly attenuated the cardio toxicity; normalized oxidative stress markers such as MDA, P.carbonyl and restored the total antioxidant capacity. SO also attenuated the increase in CAT activity. Accumulating evidence suggests that the protective effects of SO against oxidative damage could be attributed to its antioxidative properties $s^{[9,11,24]}$. The antioxidant activity of $\mathrm{SO}$ could be attributed to its phenolic lignans-type compounds namely sesamol, sesamin and sesaminol ${ }^{[6]}$. These antioxidant compounds have been found to possess antioxidant effects against oxidative damage induced by cupper in vitro study ${ }^{[10]}$ and endotoxin lipopolysaccharide $e^{[8,24]}$ and ischemiareperfusion ${ }^{[12]}$ in rats. This prevention of the DOXinduced oxidative stress damage in rats with $\mathrm{SO}$ supports the hypothesis that part of the mechanism of heart damage is attributed to the overproduction of free radicals.

In conclusion, we showed that oxidative damage to heart contributes to the myocardial toxicity induced by DOX in male rats. These effects might be limited by the use of SO. The protective effect of SO may be due to its antioxidant properties.

\section{ACKNOWLEDGEMENT}

This is a non funded project and we would like to acknowledge Department of Biology, UAEU for their full support and for making virtually all facilities available for us to use.

\section{REFERENCES}

1. Oliveira, P. J., Bjork, J. A., Santos, M. S., Leino, R. L., Froberg, M. K., Moreno, A. J., \& Wallace, K. B.2004. Carvedilol-mediated antioxidant protection against doxorubicin-induced cardiac mitochondrial toxicity Toxicol.App.Pharmacol., 200: 159-168.

2. Mukherjee, S., Banerjee, S. K., Maulik, M., Dinda, A. K., \& Maulik, K. S. 2003 Protection against acute adriamycin-induced cardiotoxicity by garlic: Role of endogenous antioxidants and inhibition of TNF- $\alpha$ expression BMC Pharmacol., 3(16): 1-9 
3. Gille, L., \& Hans, N. 1997 Analyses of the molecular mechanism of adriamycin-induced Cardiotoxicity Free Radic.Biol.Med., 23(5): 775782.

4. Abdel-Wahab, M. H., El-Mahdy, M. A., AbdEllah, M. F., Helat, G. K., Khalifa, F., \& Hamada, F. M. A. 2003 Influence of p-coumaric acid on doxorubicin-induced oxidative stress in rat's heart. Pharmacol.Res., 48: 461-465.

5. Yagmurca, M., Fadillioglu, E., Erdogan, H., Ucar, M., Sogut, S., \& Irmak, M. K. 2003 Erdosteine prevents doxorubicin-induced cardiotoxicity in rats Pharmacol.Res., 48: 377-382.

6. Suja, K. P., Jayalekshmy, A., \& Arumughan, C. 2004. Free Radical Scavenging Behavior of Antioxidant Compounds of Sesame (Sesamum indicum L.) in DPPH System J.Agric.Food.Chem., 52(4): 912-915.

7. Sankar, D., Sambandam, G., Ramakrishna Rao, M., \& Pugalendi, K. V. 2005 Modulation of blood pressure, lipid profiles and redox status in hypertensive patients taking different edible oils. Clin.Chem.Acta, 355(1): 97-104.

8. Hsu, D. Z., \& Liu, M. Y. 2002 Sesame oil attenuates multiple organ failure and increases survival rate during endotoxemia in rats. Crit.Care Med., 30(8): 1859-1862.

9. Kaur, I. P., \& Saini, A. 2000 Sesamol exhibits antimutagenic activity against oxygen species mediated mutagenicity.Mutat .Res., 470(1): 71-76.

10. Kang, M. H., Naito, M., Sakai, K., Uchida, K., \& Osawa, 2000. Mode of action of sesame lignans in protecting low-density lipoprotein against oxidative damage in vitro. Life Sci., 66(2): 161171.

11. Nakano, D., Itoh, C., Takaoka, M., Kiso, Y., Tanaka, T., \& Matsumura, Y. 2002 Antihypertensive effect of sesamin. IV. Inhibition of vascular superoxide production by sesamin. Biol.Pharmacol.Bull., 25(9): 1247-1249.

12. Utsunomiya, T., Shimada, M., Rikimaru, T., Hasegawa, H., Yamashita, Y., Hamatsu, T., Yamasaki, M., Kaku, S., Yamada, K., \& Sugimachi, K. 2003 Antioxidant and antiinflammatory effects of a diet supplemented with sesamin on hepatic ischemia-reperfusion injury in rats. Hepatogastroenterolgy, 50(53): 1609-1613.
13. Noguchi T, Ikeda K, Sasaki Y, Yamamoto J, Seki J, Yamagata K, Nara Y, Hara H, Kakuta H, Yamori Y. 2001. Effects of vitamin $\mathrm{E}$ and sesamin on hypertension and cerebral thrombogenesis in stroke-prone spontaneously hypertensive rats. Hypertens. Res. 24(6):735-42

14. Reitman, S., \& Frankel, S. 1. 1957, Determination of serum enzymes. Am. J. Clin. Path., 28: 56 -63.

15. Uchiyama, M., \& Mihara, M. 1978. Determination of malonaldehyde precursor in tissues by thiobarbituric acid test. Anal. Biochem., 86: 271278.

16. Aebi, H. 1984. Catalase in vitro, Meth Enzymol, Vol. 105: 121-126.

17. Benzie, I. F., \& Strain, J. J. 1996. The ferric reducing ability of plasma (FRAP) as a measure of "antioxidant power": the FRAP assay Anal Biochem, 293(1): 70-76.

18. Reznick, A. Z., \& Packer, L. 1994 Oxidative damage to proteins: spectrophotometric method for carbonyl assay. Metho.Enzymol., 233: 357-363.

19. Peterson, G. L. 1977 A simplification of the protein assay method of Lowry et al. which is more generally applicable. Anol. Biochem., 83: 346 356.

20. Fujimura, L., Matsudo, Y., Kang, M., Takamori, Y., Tokuhisa, T., \& Hatano, M. 2004. Protective role of $\mathrm{Nd} 1$ in doxorubicin-induced cardiotoxicity. Cardiovasc.Res., 64: 315-321

21. Dalle-Donne, I., Rossi, R., Giustarini, D., Milzani, A., \& Colombo, R. 2003 Protein carbonyl groups as biomarkers of oxidative stress. Clin.Chim.Acta, 329((1-2)): 23-38.

22. Dalloz, F., Maingon, P., Cottin, Y., Briot, F., Horiot, J. C., \& Rochette, L. 1999 Effects of combined irradiation and doxorubicin treatment on cardiac function and antioxidant defenses in the rat. Radic.Biol.Med., 26(7/8): 785-600.

23. Mizutani, H., Tada-Oikawa, S., Hiraku, Y., Kojima, M., \& Kawanishi, S. 2005. Mechanism of apoptosis induced by doxorubicin through the generation of hydrogen peroxide.Life Sci., 76: 1439-1453.

24. Hsu, D. Z., \& Liu, M. Y. 2004 Sesame oil protects against lipopolysaccharide-stimulated oxidative stress in rats. Crit.Care Med., 32(1): 227-231. 